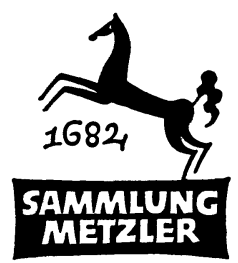

REALIEN ZUR LITERATUR

ABT. B:

LITERATURWISSENSCHAFTLICHE METHODENLEHRE 


\title{
Einführung in die Bücherkunde zur deutschen
}

Literaturwissenschaft

\author{
Mit 13 Tabellen \\ im Anhang
}

10., unveränderte Auflage

unter Mitarbeit von Werner Arnold

und Ingrid Hannich-Bode

\author{
MCMLXXXIV \\ J. B. METZLERSCHE VERLAGSBUCHHANDLUNG \\ STUTTGART
}


CIP-Kurztitelaufnahme der Deutschen Bibliothek

Raabe, Paul:

Einführung in die Bücherkunde zur deutschen Literaturwissenschaft: mit 13 Tab. im Anh. / Paul Raabe. - 10., unveränd. Aufl. / unter Mitarb. von Werner Arnold u. Ingrid Hannich-Bode. Stuttgart: Metzler, 1984.

(Sammlung Metzler; Bd. 1: Abt. B, Literaturwiss. Methodenlehre) ISBN 978-3-476-19001-7

NE: GT

Additional material to this book can be downloaded from http://extras.springer.com

ISBN 978-3-476-19001-7 ISBN 978-3-476-04154-8 (eBook) DOI 10.1007/978-3-476-04154-8

\section{1}

(C) Springer-Verlag GmbH Deutschland 1984

Ursprünglich erschienen bei J. B. Metzlersche Verlagsbuchhandlung und Carl Ernst Poeschel Verlag GmbH in Stuttgart 1961/1984 
Dieses Buch, das nunmehr in 5. Auflage erscheint, ist eine Einführung in die Praxis der Bücherkunde zur deutschen Literaturwissenschaft. Die gute Aufnahme, die das kleine Werk fand, verpflichtet den Verfasser, die Grundkonzeption von Auflage zu Auflage beizubehalten, das Neuerschienene oder Fortgeführte nachzutragen und das dadurch vielleicht Überholte zu streichen. Auch waren einige Verbesserungen und wenige Ergänzungen einzuarbeiten. Im übrigen ist die Zahl der Hilfsmittel und Nachschlagewerke bewußt knapp gehalten, so knapp, daß es dem Leser noch zumutbar ist, sich diese Titel eirzuprägen. Gerade in der in langjähriger Praxis erprobten Auswahl glaubt der Verfasser den entschiedenen Nutzen seines Unternehmens zu sehen. Die Verlockung ist groß, den Band wesentlich zu erweitern, - doch, wie gesagt, auch bei Herausgabe dieser Auflage beschränkte sich der Verfasser auf Nachträge. Eines aber mußte nunmehr in Kauf genommen werden: die Veränderungen in der Numerierung der in Text und Bibliographie genannten Werke waren nicht mehr aufzufangen, eine neue Durchzählung wurde erforderlich.

Inzwischen gab der Verfasser in der 'Sammlung Metzler c einen zweiten Band heraus, der die Darstellung der Bücherkunde in manchen Teilen fortführt: die »Quellenkunde zur neueren deutschen Literaturgeschichte «. Der Leser, der sich mit den Quellen - den Texten und Dokumenten der Dichtung - befassen will, kann dieses Werk zur Ergänzung heranziehen. Es enthält nach einer theoretischen Beschreibung der Handschriften, Drucke und Lebenszeugnisse in notwendiger Kürze Verzeichnisse der Nachlässe, Editionen und Dokumentenwerke sowie Berichte über einzelne Quelleninstitutionen.

Mit einem dritten Band gedenkt der Verfasser diese "Trilogie« literaturgeschichtlicher Hilfsmittel abzuschließen, mit einer "Bibliothekskunde«. Sie soll besonders dem Anfänger helfen, nun tatsächlich in den Besitz des gesuchten und ermittelten $\mathrm{Bu}-$ ches, Aufsatzes oder handschriftlichen Dokuments zu kommen.

Diese Bücher sind für die Wissenschaftspraxis bestimmt. Sie sollen Kenntnisse vermitteln, Realien bieten und auf diese Weise dazu beitragen, Voraussetzungen für das Studium der deutschen Literaturgeschichte $\mathrm{zu}$ schaffen.

Marbach a. N., den 15. Februar 1966

PAUL RAABE 
Die "Einführung in die Bücherkunde " hat ein ausgezeichnetes Echo und eine weite Verbreitung gefunden, so daß weitere Auflagen immer wieder notwendig werden. Dem Prinzip zurückhaltender Erweiterung bin ich auch dieses Mal treu geblieben. Freilich fordert jede Auflage eine neue Bearbeitung, um die ich in Anbetracht mehr dienstlicher und wissenschaftlicher Verpflichtungen dieses Mal Dr. Werner Arnold (Wolfenbüttel) und Ingrid Hannich-Bode (Basel) gebeten habe. Beiden möchte ich an dieser Stelle herzlich danken.

Mehr füge ich nicht an: ich hoffe, daß der Band auch weiterhin allen Hilfesuchenden die ersten Schritte erleichtert, in bibliographische Probleme einführt und so zum Fortwirken unserer Wissenschaft beiträgt.

Wolfenbüttel, im Dezember 1979 P. R. 
Vorwort zUr 5. Und 9. Auflage

EINLEITUNG . . . . . . . . . . . . . . . . . 1

A. Darstellender Teil . . . . . . . . . . . . . . . . . 4

I. Literaturwissenschaftliche Bibliographien... . . . . 4

1. Bibliographische Einführungen . . . . . . . . 5

2. Allgemeine bibliographische Werke . . . . . . . 7

3. Allgemeine periodische Bibliographien . . . . . . 14

4. Bibliographien zu einzelnen Epochen . . . . . . 18 Mittelalter - 16. Jahrhundert - Barock -

18. Jahrhundert und Goethezeit - 19. Jahrhundert Literatur seit 1880

5. Sach- und Quellenbibliographien . . . . . . . . 22

6. Bibliographien zu einzelnen Dichtern . . . . . . 26

7. Bibliographien zur vergleichenden

Literaturwissenschaft . . . . . . . . . 29 29

II. Allgemeine Bibliographien . . . . . . . . . . . 31

1. Buchveröffentlichungen . . . . . . . . . 31

2. Hochschul- und Schulschriften . . . . . . . . . 34

3. Bibliophile Drucke . . . . . . . . . . . 34

4. Zeitschriftenbeiträge, Zeitungsartikel, Rezensionen . . . . . . . . . . . . 35

5. Anonymen- und Pseudonymenlexika . . . . . 36

6. Bibliographien der Bibliographien . . . . . . . 37

III. Hilfsmittel literaturwissenschaftlicher Forschung . . . . 39

1. Handbücher und Reallexika . . . . . . . . . 39

2. Forschungsberichte . . . . . . . . . . . . . . . . 42

3. Literaturgeschichten . . . . . . . . . . . . 42

4. Tabellen- und Abbildungswerke . . . . . . . . . 45

5. Schriftstellerlexika . . . . . . . . . . . . . 46

6. Titelbücher, Roman-, Dramen-, Zitatenlexika . . . 49

7. Zeitschriften . . . . . . . . . . . . 50

IV. Allgemeine Nachschlagewerke . . . . . . . . . . . . 54

1. Allgemeinenzyklopädien . . . . . . . . . . . . 54

2. Fachenzyklopädien ... . . . . . . 56 
I. Das Bibliographieren gedruckter Quellen . . . . . 59

1. Buchveröffentlichungen . . . . . . . . . . 59

2. Zeitschriftenveröffentlichungen .. . . . . . 60

3. Zeitungsartikel . . . . . . . . . . . . . 61

II. Das Bibliographieren der Sekundärliteratur . . . . . . 62

1. Periodisches Bibliographieren . . . . . . . . 62

2. Thematisches Bibliographieren . . . . . . . 63 16. Jahrhundert - Barock -

18. Jahrhundert - Goethezeit -

19. Jahrhundert - Literatur seit 1880

3. Formales Bibliographieren . . . . . . . . . 64

Buchveröffentlichungen -

Hochschul- und Schulschriften -

Zeitschriftenbeiträge - Zeitungsartikel -

Rezensionen - Zeitschriftentitel.

III. Das Nachschlagen von Realien . . . . . . . . . 66

IV. Die Ermittlung biographischer Angaben . . . . . . . 66

C. Bibliographischer Teil . . . . . . . . . . . . . . . . 68

Verzeichnis der Bibliographien und Hilfsmittel . . . . . . . 68

Register . . . . . . . . . . . . . . . . . . . 97

ANHANG: Tabelle 1-13 